\title{
MODELO MATEMÁTICO DEL COMPORTAMIENTO MECÁNICO EN FLEXIÓN DEL CARTÓN-YESO**
}

\author{
(MATHEMATICAL MODEL OF THE MECHANICAL BEHAVIOUR OF GYPSUM \\ PLASTERBOARD IN BENDING)
}

\section{L. de Villanueva, F. Lasheras, M. del Río y F. Hernández-Olivares \\ Departamento de Construcción y Tecnología Arquitectónicas. ETS de Arquitectura}

Fecha de recepción: 29 - III - 94

Universidad Politécnica de Madrid**

ESPAÑA

\section{RESUMEN}

Se presenta un modelo matemático que predice la carga de rotura de las placas de cartón-yeso en ensayos a flexotracción, a partir del conocimiento de sus dimensiones geométricas y de las propiedades mecánicas de los materiales componentes. Los valores asi obtenidos se comparan con los que proponen diversas normas europeas y americanas para la carga de rotura mínima, asi como con medidas experimentales en laboratorio,

obteniéndose en ambos casos un acuerdo excelente.

\section{SUMMARY}

The article proposes a mathematical model which predicts the bearing load capacity of gypsum plasterboard sheets in bending tests, based on the geometric dimensions and the mechanical properties of the constituent materials. Thus obtained values are compared with those proposed by various European and American Standards for the minimum bearing load capacity as well as with experimental laboratory measurements. In both cases the results obtained reached an excellent level of agreement.

\section{Introducción}

Como es bien sabido, un sandwich es una placa formada básicamente por un elemento central, llamado núcleo, y dos láminas exteriores delgadas o gruesas, llamadas pieles, perfectamente adheridas a las caras del núcleo. La función principal de las pieles es resistir los esfuerzos de tracción y proteger al núcleo, que aporta — sobre todo— rigidez y baja densidad al compuesto, además de resistencia al esfuerzo cortante (Fig. 1).

Las placas de cartón-yeso, de amplio y conocido uso en construcción, se componen de un núcleo grueso de yeso y dos pieles delgadas de cartón adheridas a las caras anterior y posterior del yeso.

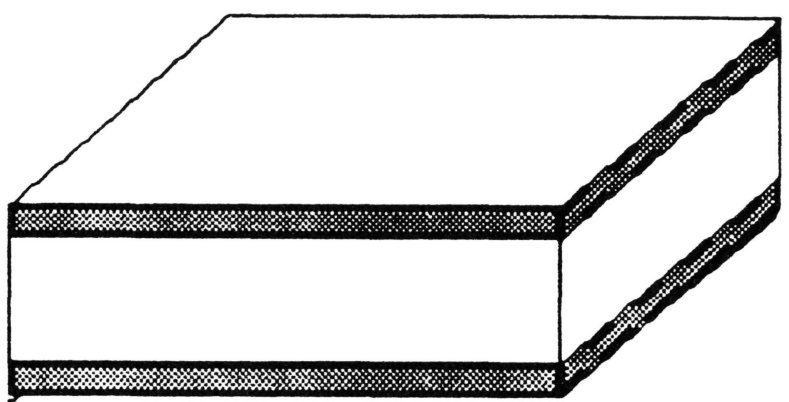

Fig. 1. Placa sanduich

El núcleo de yeso se produce básicamente a partir de semihidrato con pequeñas adiciones de otros productos, entre los que destaca el almidón, cuya función principal es retener el agua a disposición del yeso durante su fraguado.

* Conferencia en el XX Congreso de Eurogypsum, Estocolmo 1994.

** Trabajo realizado dentro del convenio de colaboración entre Española de Placas de Yeso. S.A. —EPYSA—y el citado Departamento universitario. 
Cada una de las pieles de cartón se compone de varias láminas delgadas de papel reciclado (de cinco a nueve). Las láminas están impregnadas de resina, salvo la interior en contacto con el yeso que se trata con polímeros del tipo silenos siloxanos. Este último ensimaje, que repele el agua líquida, recubre las fibras de celulosa sin cerrar totalmente el espacio entre fibras. Así, pues, las láminas interiores de papel contribuyen a retener parte del agua necesaria de fraguado y facilitan la eliminación del vapor de agua a través de los espacios entre fibras, promoviendo una adherencia perfecta entre el cartón y el yeso sin que se produzca estratificación en el yeso cerca de la intercara, con posibles recalcinaciones locales en el secado.

La resistencia a tracción del cartón de las pieles - con esta textura y tratamiento- y su módulo de Young son mucho mayores que los del yeso, lo que permite afirmar que las placas de cartón-yeso reúnen las características básicas de un elemento sandwich ${ }^{1}$.

\section{Objetivos}

El objetivo principal de este trabajo ha sido elaborar un modelo del comportamiento mecánico de las placas de cartón-yeso sometidas a flexotracción, del que pueda deducirse una expresión matemática para predecir la carga de rotura en función de las características del ensayo, propiedades de los materiales que componen la placa y dimensiones de las placas ensayadas.

Dos razones hacían necesario este modelo. La primera de índole científica: conocer los mecanismos que regulan las propiedades mecánicas de las placas. La segunda de alcance pragmático: las normas sobre este producto — vigentes en varios países (ASTM, BS, DIN, NF, ONORM, UNE) internacionales (ISO) o en fase de elaboración (proyecto CEN) 2establecen valores mínimos exigibles de la carga de rotura de las placas de cartón-yeso, en sentido paralelo y transversal al de la laminación, ensayadas a flexión en tres puntos, en condiciones controladas de laboratorio y para espesores normalizados.

Estos valores numéricos son consecuencia de gran número de medidas experimentales en laboratorio, pero varían algo entre sí los propuestos por las diferentes normativas, al no responder a un patrón racional o modelo matemático aceptado universalmente 3
Así pues, una relación matemática simple, basada en un modelo de las placas de cartón-yeso, puede contribuir a facilitar la adopción de unos criterios mayoritariamente aceptables respecto de la resistencia mecánica exigible a las placas.

\section{Metodología}

Una vez establecidas las características básicas de la estructura de las placas de cartón-yeso y las propiedades de los materiales constituyentes, se ha elaborado teóricamente un modelo matemático de su comportamiento mecánico a flexotracción. Este modelo proporciona una sencilla relación entre la carga de rotura de las placas, las propiedades mecánicas de los materiales que la forman, las dimensiones y la separación entre apoyos en los ensayos a flexión.

Verificada la consistencia interna de la relación matemática obtenida, se realizó una serie programada de ensayos de rotura a flexotracción, variando las dimensiones (anchura y espesor) de las placas, la separación entre apoyos y la posición de la cara de la placa en tracción o en compresión arriba o abajo, obteniéndose los valores de las cargas de rotura. Algunos de estos valores - los realizados con probetas de dimensiones normalizadas- se comparan con los mínimos establecidos por las normas europeas.

A continuación, se determinaron experimentalmente las propiedades mecánicas del yeso y del cartón necesarias para aplicar la expresión matemática y se comparan las predicciones del modelo con los valores de la carga de rotura obtenida en los ensayos.

Finalmente, se comparan los valores mínimos de las cargas de rotura establecidos por las normas ASTM y europeas con las predicciones del modelo.

\section{Modelo matemático}

Se propone un modelo matemático, para describir el comportamiento mecánico a flexotracción de las placas de cartón-yeso, basada en la teoría de la sección transformada.

De acuerdo con esta teoría el análisis del comportamiento mecánico de la placa "s̄andwich" se realiza obteniendo en primer lugar las propiedades de la sección transformada mediante la aplicación de las siguientes reglas : 
1. Se selecciona una placa de longitud 1, espesor t y anchura b.

2. Se elige un módulo elástico de referencia, por ejemplo el de una de las caras. En este caso se adoptará más adelante el de la lámina de cartón de la cara de la placa que está solicitada a tracción, $\mathrm{E}_{\mathrm{C}}$.

3. Se obtiene la razón modular $n$ de cada componente de la sección, definido como el cociente entre su módulo de elasticidad $\mathrm{E}_{\mathrm{i}} \mathrm{y}$ el que se ha tomado como referencia:

$$
\mathrm{n}_{\mathrm{i}}=\frac{\mathrm{E}_{\mathrm{i}}}{\mathrm{E}_{\mathrm{c}}}
$$

4. Las dimensiones de la sección transformada se obtienen multiplicando la anchura de la sección real por la razón modular de cada componente.

5. La adherencia entre el núcleo y las láminas se considera perfecta.

A continuación, se aplica a la sección transformada la teoría elemental de vigas con sección de anchura variable, con los matices que se detallan más adelante.

En cuanto a las propiedades de la sección transformada y de la sección real debe tenerse en cuenta que:

- La deformación unitaria es la misma en la sección transformada que en la sección real.

- La tensión en la transformada es ficticia. Se trata de una pseudotensión. La tensión verdadera en la sección real se obtiene multiplicando la pseudotensión por la razón modular correspondiente.

- El esfuerzo cortante se calcula sobre la sección real teniendo en cuenta la anchura verdadera del núcleo, que es quien aporta la mayor contribución.

Establecidas las bases de la teoría de la sección transformada se describe su aplicación al caso de una placa de cartón-yeso sometida a flexotracción de ensayo a tres puntos.

Se supone una placa de cartón-yeso similar a la que se muestra en la Fig. 4, de dimensiones 1 x b x t (longitud $\mathrm{x}$ anchura $\mathrm{x}$ espesor). Se denomina $\mathrm{c}$ al espesor de cada una de las pieles de cartón, y d al espesor del núcleo de yeso, esto es:

$$
\mathrm{t}=2 \mathrm{c}+\mathrm{d}
$$

(c) Consejo Superior de Investigaciones Científicas Licencia Creative Commons 3.0 España (by-nc)
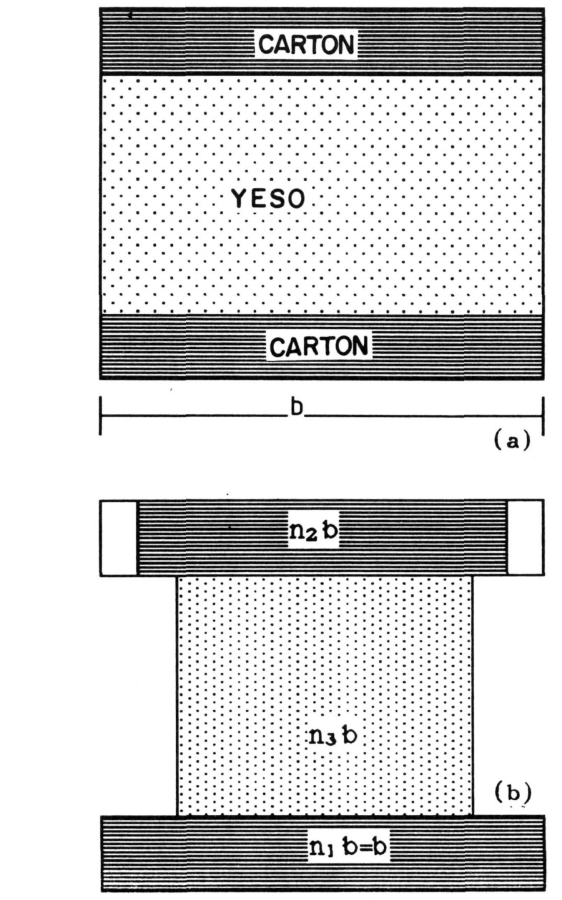

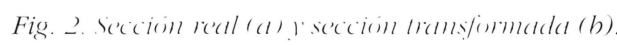
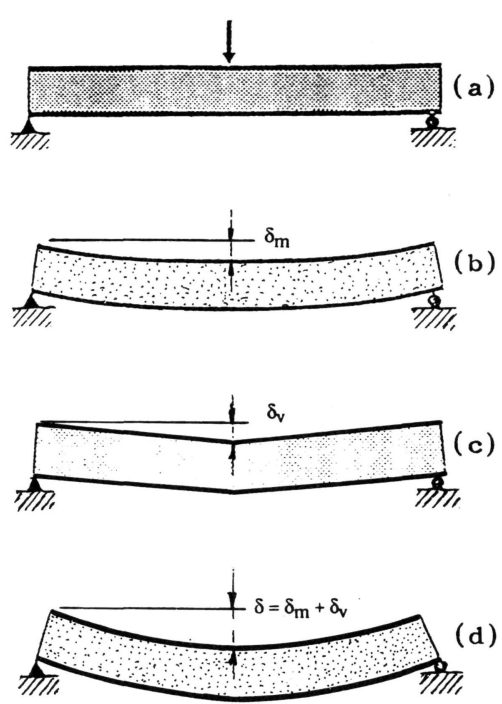

Fig. 3. Esquema del ensayo a flexión en tres puntos de una viga tipo sandwich

(a) Esquema general. (b) Flecha debida a la flexión primaria. (c) Flecha debida al cortante en el núcleo. (d) Acción combinada de la flecha y el cortante.

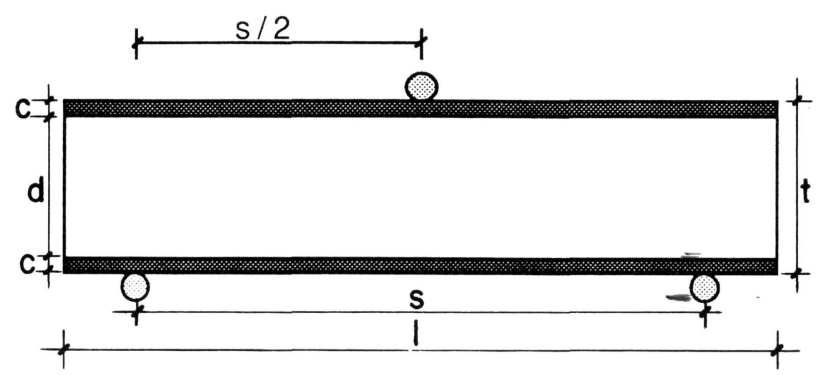

Fig. 4. Esquema del ensayo para placas de cartón-yeso.

http://informesdelaconstruccion.revistas.csic.es 
El análisis posterior se basa en las hipótesis siguientes:

- El núcleo de yeso es rígido, no flexible.

- No tienen importancia los efectos viscoelásticos.

- Se desprecia el peso de la placa ?

\subsection{Análisis de la flecha máxima}

La Fig. 3a muestra un esquema del ensayo a flexión simple (tres puntos) de una viga sandwich. La Fig. $3 b$ es un esquema de la flecha máxima debida a la flexión primaria de la sección transformada. En la Fig. 3c se muestra la flecha debida al cortante en el núcleo. Por último, la Fig. 3d muestra la acción combinada.

Este modelo representa adecuadamente el comportamiento a flexión de las placas de cartón-yeso con un núcleo rígido de yeso al que se adhieren perfectamente las pieles de cartón. Así, pues, la flecha total se obtiene sumando la debida a la sección transformada y la debida al cortante en el núcleo (Fig. 3):

$$
\delta=\delta_{\mathrm{m}}+\delta_{\mathrm{v}}
$$

Designando por s a la separación entre apoyos, la flecha total es la suma de la flecha debida a la flexión y la debida al cortante, y tiene la siguiente expresión:

$$
\delta=\frac{P s^{3}}{48 \mathrm{E}_{C} \mathrm{I}}+\frac{P s}{4 \mathrm{~A}_{\mathrm{V}} \mathrm{G}}
$$

siendo P la carga aplicada, $\mathrm{E}_{\mathrm{C}}$ el módulo de Young del cartón traccionado (corresponde a la fibra extrema), I la inercia de la sección transformada y $A_{v} G$ la rigidez a cortante: área de la sección transversal d x b multiplicada por el módulo de rigidez a cortadura, G, del yeso.

\subsection{Análisis del comportamiento mecánico de la placa sometida a flexotracción}

El módulo resistente $\mathrm{W}$ de la sección transformada se relaciona con el momento flector $\mathrm{M}$ y la tensión en la fibra extrema $\sigma$ mediante la expresión:

$$
\mathrm{W}=\frac{M}{\sigma}
$$

Se aceptará como criterio de rotura de la placa el de la fractura a tracción de la lámina de cartón, con independencia de que el núcleo de yeso sufra fisuración previa, ya que en cualquier caso continúa aportando inercia a la sección mientras el cartón no se fracture.

(c) Consejo Superior de Investigaciones Científicas Licencia Creative Commons 3.0 España (by-nc)
Así pues, $\sigma_{\max }=\sigma_{c \text {, placas }}$, donde $\sigma_{\text {c.placas }}=\lambda \cdot \sigma_{c}$, siendo $\lambda$ el factor de reducción de la resistencia a tracción del cartón $\sigma_{c}$, como consecuencia del proceso de fabricación de las placas, $\lambda \approx 0,5$. De acuerdo con el criterio de rotura que se ha adoptado, la carga de rotura a flexotracción se obtiene a partir del momento máximo:

$$
M_{\max }=\frac{P S}{4}
$$

Como

$$
\mathrm{M}_{\max }=\mathrm{W} \sigma_{\max }
$$

Resulta

$$
\mathrm{P}=\frac{4 \mathrm{~W} \sigma_{\max }}{s}=\frac{2 \mathrm{~W} \sigma_{\mathrm{c}}}{\mathrm{s}}
$$

\subsection{Obtención del momento de inercia de la sección}

Sea I el momento de inercia de la sección transformada, alrededor de un eje perpendicular al eje neutro y contenido en el plano de simetría de la placa, su plano neutro. Evidentemente,

$$
\mathrm{I}=2 \mathrm{I}_{\mathrm{C}}+\mathrm{I}_{y}
$$

donde $\mathrm{I}_{c}$ es el momento de inercia de la sección de cada una de las láminas de cartón e $\mathrm{I}_{Y}$ el del alma de la sección transformada, correspondiente al núcleo de yeso.

Teniendo en cuenta que $c<<$, puede hacerse la siguiente aproximación:

$$
\mathrm{d} \approx \mathrm{t}
$$

De este modo, el momento de inercia de la sección transformada resulta

$$
\mathrm{I}=\frac{\mathrm{bc} \mathrm{c}^{3}}{6}+\frac{\mathrm{bct}^{2}}{2}+\frac{\mathrm{nbt}^{3}}{12} \approx \frac{\mathrm{bct}^{2}}{2}+\frac{\mathrm{nbt}^{3}}{12}
$$

donde $\mathrm{n}$ es el cociente entre el módulo de Young del yeso y el del cartón en la cara traccionada.

Por otro lado, el módulo resistente de la sección es:

$$
W=\frac{2 I}{t}
$$


Luego, sustituyendo la expresión obtenida para I:

$$
\mathrm{W}=\mathrm{bct}+\frac{\mathrm{nbt}^{2}}{6}
$$

Sustituyendo la expresión obtenida para W, resulta

$$
\mathrm{P}=\sigma_{\mathrm{c}} \frac{2 \mathrm{bct}}{\mathrm{s}}\left(1+\frac{\mathrm{nt}}{6 \mathrm{c}}\right)
$$

En esta fórmula aparecen claramente diferenciadas las contribuciones de las láminas de cartón y del núcleo de yeso a la resistencia a flexotracción del panel sandwich. En efecto, la lámina de cartón solicitada a tracción soporta una carga en rotura:

$$
P_{c}=\sigma_{c} \frac{2 b c t}{s}
$$

mientras que la del núcleo de yeso es:

$$
\mathrm{P}_{\mathrm{y}}=\sigma_{\mathrm{c}} \frac{\mathrm{nbt}^{2}}{3 \mathrm{~s}}
$$

independiente del espesor de las láminas de cartón c.

Del trabajo de H. G. Allen ya citado (cfr. ref. 1, página 2) de los valores prescritos en la norma ASTM, se desprende que hay un modelo matemático más sencillo utilizado en esos documentos, en el que se desprecia la aportación del núcleo de yeso a la resistencia del sandwich. Sólo se considera la resistencia del cartón y se desprecia el segundo término de la expresión matemática del modelo expuesto, que hace referencia al núcleo de yeso en sus dimensiones y en el valor relativo de su módulo de Young. Esta simplificación, que en la práctica desprecia las características del yeso, introduce desviaciones de cierta importancia entre los valores teóricos y los experimentales. Por ello, la principal aportación del presente trabajo, en lo relativo al modelo matemático, es el poder estimar, de modo sencillo, la aportación del núcleo de yeso a la resistencia a flexión del conjunto.

\section{Comparación entre medidas experimentales y los valores que predice el modelo}

Las placas de cartón-yeso "ensayadas en laboratorio han sido suministradas por EPYSA, en tres espesores diferentes: 9,5, 12,5 y $15 \mathrm{~mm}$, respectivamente.
Tras su recepción se han cortado para ser ensayadas a flexotracción, en tres conjuntos de distinta anchura (200, 300 y $400 \mathrm{~mm}$ ). De cada uno de estos conjuntos se han preparado grupos de probetas de distinta longitud, con el fin de disponer de probetas que pudieran ensayarse variando la separación entre apoyos (250, 350 y $450 \mathrm{~mm}$, respectivamente).

Todas estas manipulaciones relativas a la preparación de probetas y ensayos a flexotracción de la placas se han realizado siguiendo las indicaciones de las Normas UNE 102023 (Placas de cartón-yeso. Condiciones generales y especificaciones) y UNE 10035 (Placas de cartón-yeso. Métodos de ensayo), que coinciden básicamente con las que recoge la ISO 6308 (Gypsum plasterboard-Specification, 1980) y el Proyecto CEN (versión de 1989).

Sin embargo, también se han preparado conjuntos de probetas de dimensiones no contempladas en la normativa citada anteriormente ni en la norma ASTM (C 36-80: Standard Specification for Gypsum Wallboard) correspondiente, con el fin de analizar la variación de la carga de rotura con la anchura de las probetas y de la separación entre apoyos, y corroborar así la validez del modelo.

Al mismo tiempo se tomaron 18 muestras de la pasta de yeso de la misma amasada de las placas en moldes de 40x40x160 mm (antes y durante el vibrado), con las que se determinaron el módulo de rotura del material del núcleo, su resistencia a flexión y su resistencia a compresión, entre otros datos.

Las propiedades mecánicas del cartón se midieron (UNE 57028; ISO 1924) ensayando a tracción probetas obtenidas de los mismos rollos con los que se fabricaron las placas de cartón-yeso ensayadas. A partir de estos ensayos se determinó el límite elástico y el módulo de Young del cartón.

Estas propiedades del yeso del núcleo y del cartón de las pieles se han utilizado en la fórmula del modelo matemático junto con las dimensiones de las probetas de las placas, obteniéndose en cada caso un valor teórico de la carga de rotura de la placas a flexión.

En las Figs. 5, 6 y 7 se comparan los valores experimentales de las cargas de rotura con las dēl modelo matemático para espesores de 9,5, 12,5 y $15 \mathrm{~mm}$, respectivamente, variando la separación entre apoyos y la anchura de las placas ensayadas. 


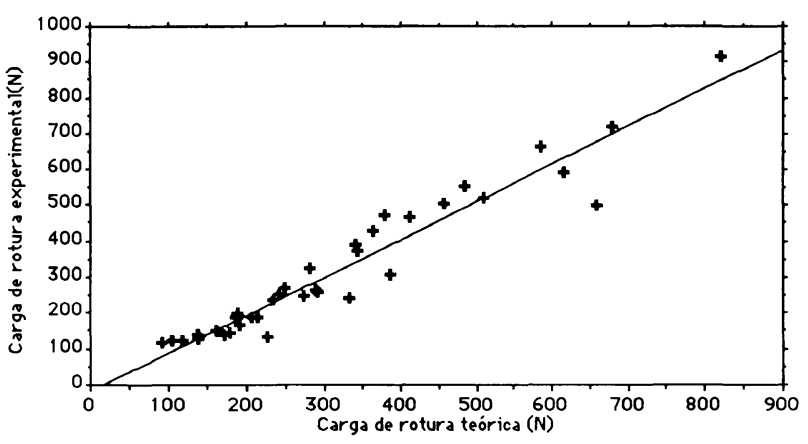

Fig. 5. Regresion entre las cargas de rolura leóricas y' experimentales (placas de 9,5 $\mathrm{mm}$ de espesor) $P_{\text {exp }}=1,05 P_{\text {terim }}-18,6(\mathrm{~N})$.

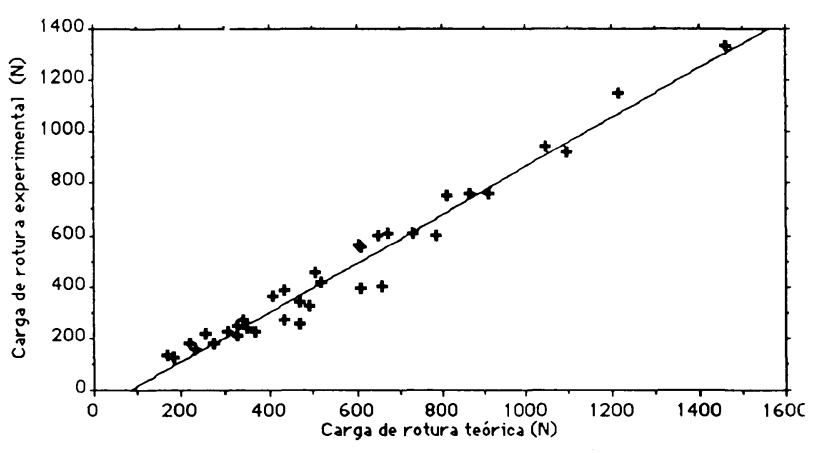

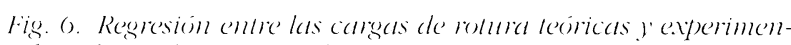
tales (placas de $12,5 \mathrm{~mm}$ de espesor) $P_{\text {exp }}=0,95 P_{\text {teorr }}-78,6(\mathrm{~N})$.

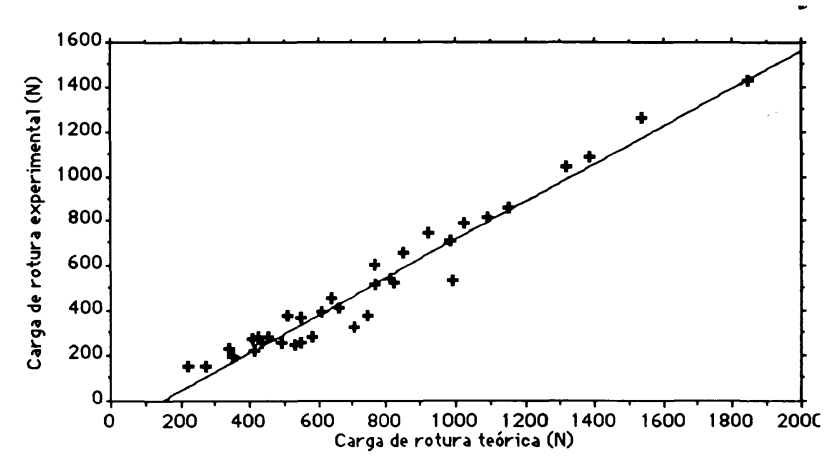

Fig. 7. Regresion entre las cargas de rolura teriticas y experimentales (placas de $15 \mathrm{~mm}$ de espesor) $P_{\text {exp }}=0,84 P_{\text {terir. }}-122,7(\mathrm{~N})$.

\section{Discusión. Análisis de los valores de la carga de rotura propuestos por la normativa actual}

Del examen de las figuras anteriores se desprende que el modelo matemático desarrollado describe muy bien el comportamiento mecánico de las placas de cartón-yeso en ensayos a flexotracción, que son los exigidos por la normativa para verificar sus propiedades mecánicas.

Este modelo puede utilizarse como referencia para calibrar la calidad del producto e incidir en el pro- ceso de fabricación, sobre aquellas variables que puedan estar alterando las prestaciones previstas.

Los valores teóricos de la carga de rotura se basan en unos datos geométricos (las dimensiones de las placas) y unas propiedades mecánicas de los componentes: el cartón y el yeso. Los primeros son únicos y no admiten variación: se utilizan en el modelo teórico los mismos que caracterizan a las placas que se ensayan.

Sin embargo, los segundos pueden variar. Al no poder extraer el cartón de una placa elaborada sin deteriorarlo, los ensayos mecánicos para determinar su límite elástico y el módulo de Young se han realizado sobre probetas de cartón que no han sufrido las acciones químicas, higrotérmicas y mecánicas del proceso de fabricación de las placas.

Igualmente, el fraguado y secado del yeso de las placas se realiza dentro del proceso de fabricación, mientras que el de las probetas prismáticas extrádas para ensayo de caracterización mecánica han fraguado en condiciones óptimas de laboratorio.

Esto explica, sin duda, las pequeñas diferencias entre el conjunto de valores teóricos —en placas gruesas, especialmente- y experimentales, pudiendo afirmarse que el proceso de fabricación es excelente, ya que el modelo matemático proporciona los valores óptimos de las cargas de rotura.

Existe, sin embargo, un sesgo en las placas de mayor grosor hacia valores experimentales inferiores a los que predice el modelo, que puede analizarse a la luz de los valores mínimos de la carga de rotura que establecen las diversas normativas.

La Fig. 8 muestra un diagrama tridimensional de los valores de la carga de rotura mínimos exigidos por las Normas UNE, ISO y Proyecto CEN, de probetas ensayadas en la dirección longitudinal de las fibras que componen las láminas de cartón.

Se han agrupado estos valores porque todos ellos se basan en el mismo tipo de probetas (400x300xt mm) y separación entre apoyos $(350 \mathrm{~mm})$. El Proyecto CEN es el único que prevé placas de espesores superiores a $18 \mathrm{~mm}$, y establece un incremento de la carga límite inferior proporcional al espesor, con una pendiente inferior al aumento común establecido por las tres normas citadas para las placas de menor espesor. 


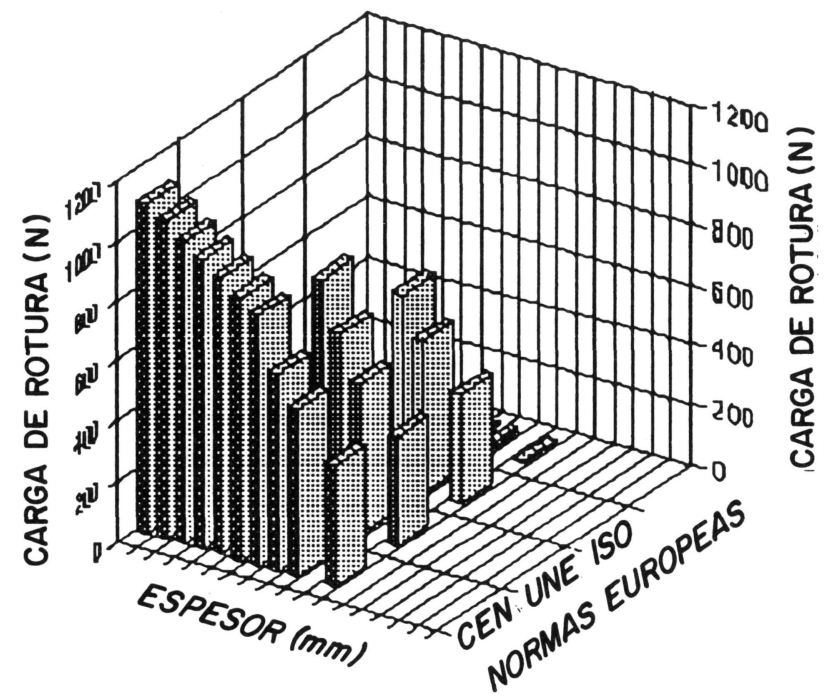

Fig. 8. Diagrama tridimensional de las cargas de rotura longitudinales establecidas por las Normas ISO, UNE y Proyecto CEN, para distintos espesores de placas de anchura normalizada.

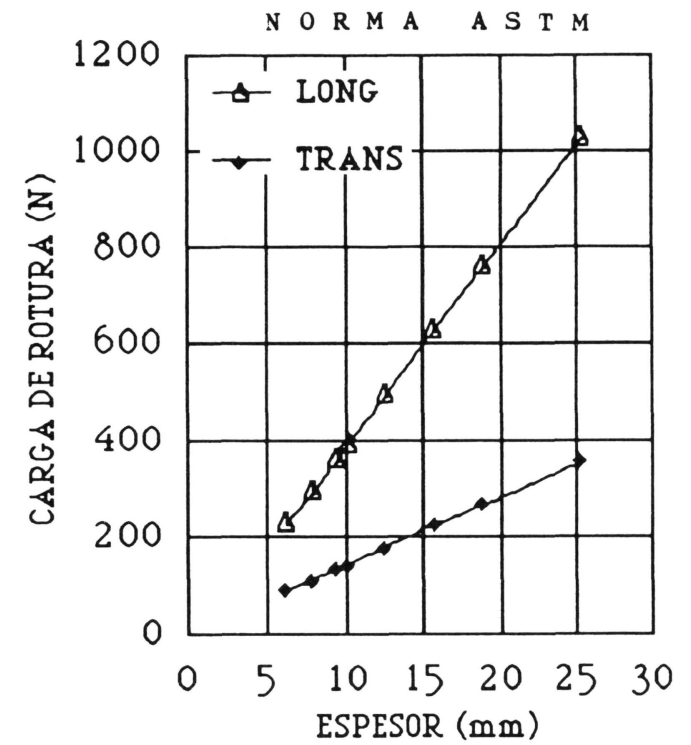

Fig. 10. Valores minimos de la carga de rolura establecidos por la Norma ASTM.

En cambio, la Norma ASTM (Fig. 10) establece una relación estrictamente lineal para todo tipo de espesores. Las probetas son de dimensiones ligeramente diferentes $(405 \times 305 \times \mathrm{mm})$ a las determinadas por las normas europeas. Como también lo es la separación entre apoyos $(356 \mathrm{~mm})$. Esto excluye la comparación directa entre los valores ASTM y los europeos.

En la Fig. 11 se han representado los resultados experimentales de seis conjuntos de ensayos de (c) Consejo Superior de Investigaciones Científicas Licencia Creative Commons 3.0 España (by-nc)

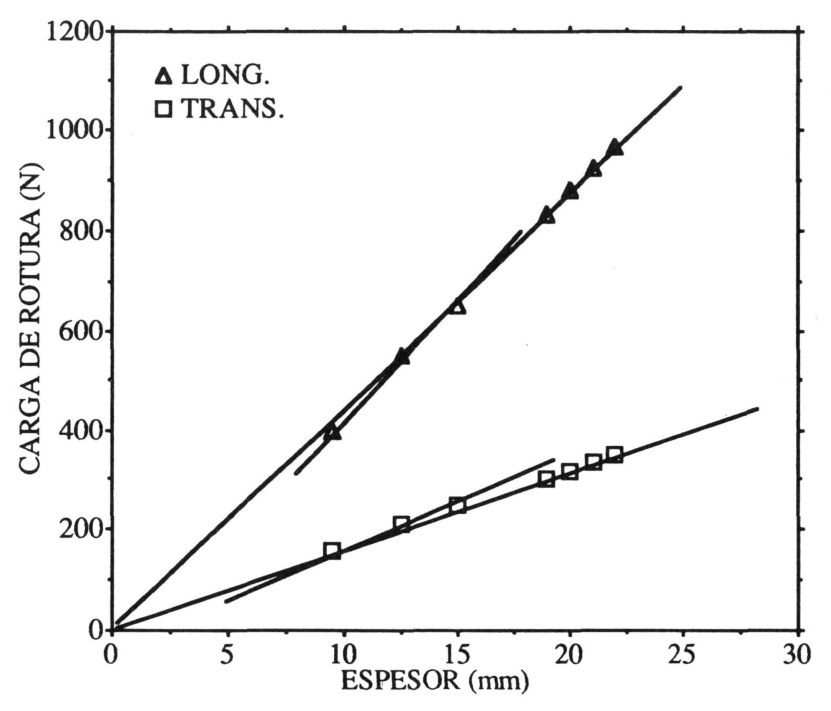

Fig. 9. Cargas de rotura establecidas en el proyecto CEN.

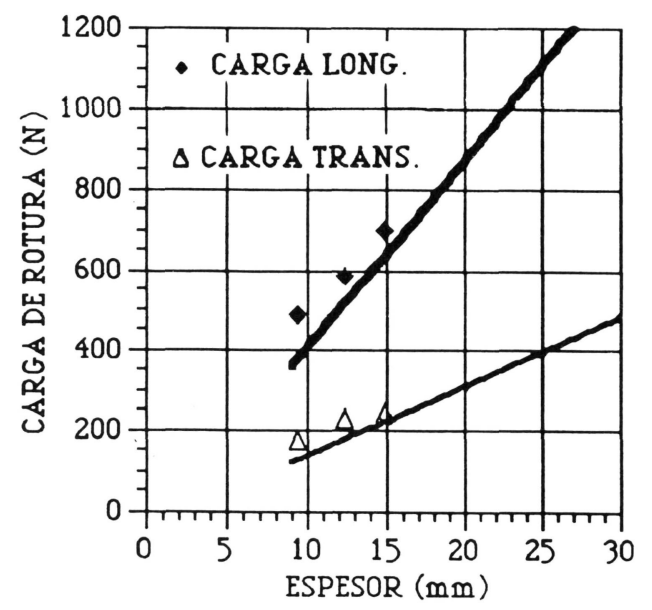

Fig. 11. Cargas de rolura de seis conjunlos de medidas experimentales (ensayo longitudinal y transversal). Las lineas representan los valores mínimos de las cargas de rotura prescritos por la normativa europea.

rotura de placas realizados de acuerdo con las prescripciones UNE en cuanto a dimensiones, separación entre apoyos, velocidad de carga y preparación de las muestras. Las líneas representan los valores mínimos que establece el conjunto de las normas europeas. Se aprecia que el comportamiento del material es plenamente satisfactorio.

Así, pues, se destaca que el modelo matemático elaborado da razón de los valores numéricos propuestos por la normativa internacional, por lo que pro- 
cedería su inclusión en las citadas Normas, al menos para justificar los valores numéricos establecidos.

Una verificación destacable de lo que se afirma en el párrafo anterior se encuentra en el análisis del factor de conversión establecido en las Normas ISO y Proyecto CEN, cuando se desea obtener la carga de rotura mediante ensayos a flexión de probetas de $400 \mathrm{~mm}$ de anchura y razón de esbeltez 40:1 (relación entre la separación entre apoyos y el espesor):

$$
\mathrm{f}=\frac{400 \times 350}{300 \times s}=\frac{466,7}{s}
$$

Este factor se obtiene directamente de la fórmula derivada con el modelo ${ }^{?}$.

Por último, la relación lineal ${ }^{*}$ —o lineal a tramosentre el espesor y la carga de rotura implica, según el modelo elaborado, una variación de la relación entre módulos de Young del yeso y del cartón con el espesor de las placas. Esta variación es consecuencia necesaria del proceso de fabricación de las placas, y utilizando los valores mínimos de la resistencia a tracción del cartón exigidos por la norma (22 kg en dirección longitudinal, $6,5 \mathrm{~kg}$ en dirección transversal), se obtiene - para las Normas europeas-:

$$
\mathrm{n}_{\mathrm{LONG}}=\frac{1,40}{\mathrm{t}} ; \mathrm{n}_{\mathrm{TRANS}}=\frac{2,15}{\mathrm{t}}
$$

En cualquier caso, al tratarse de un modelo predictivo, la aplicación de la fórmula obtenida para la carga de rotura $\mathrm{P}$ requiere la obtención previa, en fábrica o en laboratorio, de los parámetros característicos de las propiedades de los materiales constituyentes, particularmente la relación modular $\mathrm{n}$ y el factor $\lambda$ entre la resistencia a tracción del cartón antes y después de sufrir el proceso higrotérmico y mecánico de la fabricación de las placas (en esta investigación, $\lambda=0,5$ ). Después, la obtención experimental de al menos un valor de la carga de rotura para placas de geometría determinada, aseguraría la precisión del modelo para las cargas de rotura del resto de las geometrías y separación entre apoyos.

Los valores de la Norma ASTM llevan también a relaciones de este tipo. Sin embargo, si se hace un análisis exponencial se obtienen relaciones que proporcionan los siguientes valores para espesores crí- ticos (decaimiento de 1/e): $14 \mathrm{~mm}$ dirección transversal, $19 \mathrm{~mm}$ dirección longitudinal, muy de acuerdo con los límites usuales en la fabricación de las placas.

Para estudiar la variación de la rigidez del núcleo de yeso con el espesor de las placas, dentro del mismo proceso de fabricación, se han hecho medidas de la dureza Shore $\mathrm{C}$ del yeso del núcleo y de 18 probletas de yeso extraídas del vibrador antes de pasar por los rodillos de laminación, obteniéndose los siguientes valores:

Dureza Shore C

Yeso extraído del vibrador 62 Yeso del núcleo (placas 9,5 mm espesor) 60 Yeso del núcleo (placas 12,5 mm espesor) 54 Yeso del núcleo (placas $15 \mathrm{~mm}$ espesor) 53

que confirman el pequeño deterioro de las propiedades mecánicas del núcleo de yeso para las placas de mayor grosor.

\section{Conclusiones}

1. Se ha desarrollado un modelo matemático del comportamiento mecánico de las placas de cartónyeso, basado en la teoría de la sección transformada de paneles "sandwich", que proporciona la carga de rotura de las placas en ensayos a flexión en función de las propiedades mecánicas de los materiales constituyentes, dimensiones de las placas, separación entre apoyos y arquitectura interna de las placas.

2. Los ensayos experimentales realizados en laboratorio confirman la validez del modelo, al menos para placas de espesores 9,5, 12,5 y $15 \mathrm{~mm}$. Sin embargo, algunas placas del mayor espesor tienen una resistencia a rotura inferior a la que predice el modelo, lo que sugiere que el proceso de fabricación de placas gruesas altera la relación entre módulos $\mathrm{n}$, haciéndola menor que para placas delgadas, como confirman medidas de dureza Shore C del yeso del núcleo de las placas.

3. Los valores mínimos de la carga de rotura establecidos en las diversas normativas europeas y ASTM siguen leyes de evolución lineal con el espesor de las placas, lo cual imptica - a la luz del modelo elaborado- que aceptān que el núcleo de yeso no alcance su rigidez óptima en placas de espesor superior a $15 \mathrm{~cm}$. 
4. La relación matemática obtenida debe incorporarse a la normativa actual, con el fin de que los valores propuestos para las cargas mínimas de rotura de las placas se basen en argumentación científica y los factores de corrección aceptados si se modifica la separación entre apoyos se entiendan con fundamento analítico.

\section{Agradecimientos}

Esta investigación forma parte del Convenio de Colaboración Científica y Técnica entre el Departamento de Construcción y Tecnología Arquitectónica de la Universidad Politécnica de Madrid y Española de Placas de Yeso, S.A. (EPYSA), y ha sido financiada con cargo a dicho Convenio de Colaboración.
Se agradece al Departamento de I+D de EPYSA la colaboración prestada y sus valiosas aportaciones en el suministro de documentaciones, material y discusiones sobre lo anteriormente expuesto, así como su autorización para hacer públicos los resultados contenidos en esta conferencia.

Se agradece a Ernesto A. Rodríguez, becario de investigación, y Fernando Egido, técnico de laboratorio, su valiosa colaboración en la realización del trabajo.

Finalmente, a la Eurogypsum se la agradece la ocasión que nos ha ofrecido, en el marco de su XX Congreso, para poder exponer la presente conferencia.
${ }^{1}$ H. G., Allen. "Analysis of Structural Sandwich Panels", Pergamon Press, 1969.

2 ASTM C 473-81. "Standard Methods for Physical Testing of Gypsum Board Products, Gypsum Lath, Gypsum Partition, Gypsum Slabs." C 36-80. "Stardard Specification for Gypsum Wallboard", Ed. American Society for Testing and Materials, 1983.

BS 1230. Part 1, 1985: Gypsum plasterboard. Part. 1. Specification for plasterboard excluding materials submitted to secondary operations, Brittish Standard Institution, 1985.

DIN 18 180. Gipskartonplatten. Arten, Anforderungen, Prüfung. Deutsches Institut für Normung, Dezember, 1987.

NF P 72-302. Plaques de parement en plâtre. Définition, spécifications et essais. Association française de normalisation (Afnor), Octobre, 1981.

ONORM B 3410. Gipskartonplatten. Arten, Anforderungen, Prüfungen. Osterreiches Normungsinstitut (ON), Dezember, 1976.

UNE 102-023-83. Placas de cartón-yeso. Condiciones generales y especificaciones. UNE 102-035-83. Placas de cartón-yeso. Métodos de ensayo, Asociación española de normalización (Aenor), 1983.

ISO 0308. "Gypsum Plasterboard — Specification - International Organization for Standardization. Ist. Ed., 1980-11-01.

DRAFT Proposal for CEN Standard. "Gypsum Plasterboard Specification-Test Methods. June, 1989.

3 Ver anexo con los valores de las normas.

${ }^{4}$ Richard E., Chambers. "Flat Sandwich Structures", in Structural Plastics Design Manual, Ch. 8. ASCE Manuals and Reports on Engineering Practice, N.* 63, 1984.

5 Quizá a este supuesto se deba un pequeño desajuste observado entre el modelo teórico y los resultados experimentales.
De todos modos el error es pequeño y se prefiere mantener esta hipótesis en beneficio de la sencillez.

6 Se utilizan placas de cartón-yeso normalizadas según UNE 102023-83, equivalentes a las plaques de parament de la NF P 72302, a las Gypsum Wallboard de la BS 1230: Part 1:1985, y a las Gipskartonplatten de DIN 18.180, ISO 6308-1980 tipo 4.1.1 y prEN 26.308 tipo 4.1.1.

7 En efecto, como

$$
\mathrm{P}=\sigma_{\mathrm{c}} \frac{2 \mathrm{bct}}{\mathrm{s}}\left(1+\frac{\mathrm{nt}}{\sigma_{\mathrm{c}}}\right)
$$

si $s=350$ y b-300, se obtiene para la carga de rotura

$$
\mathrm{P}=\sigma_{\mathrm{c}} \frac{2300 \mathrm{ct}}{350}\left(1+\frac{\mathrm{nt}}{6 \mathrm{c}}\right)
$$

ahora bien, para una razón de esbeltez 40:1, se tiene

$$
\frac{400}{300}=\frac{\mathrm{s}}{350} \text {, luego } \mathrm{P}_{\mathrm{s}}=\sigma_{\mathrm{c}} \frac{2400 \mathrm{ct}}{\mathrm{s}}\left(1+\frac{\mathrm{nt}}{6 \mathrm{c}}\right)
$$

Luego, el factor $\mathrm{f}_{\mathrm{s}} \mathrm{P}_{\mathrm{s}}=$ f.P, se obtiene dividiendo $\mathrm{P}_{\mathrm{s}}$ entre $\mathrm{P}$, esto es

$$
\mathrm{f}=\frac{400 \times 350}{300 \times \mathrm{s}}=\frac{466,7}{\mathrm{~s}}
$$

${ }^{8}$ En ASTM es $\mathrm{P}_{\text {TRANS }}=14 \mathrm{t}$ y $\mathrm{P}_{\text {LONG }}=42 \mathrm{t}-42=42(\mathrm{t}-1)($ para $\mathrm{P}$ en $\mathrm{N}$ y t en $\mathrm{mm}$ ). Los valores contenidos en prEN parecen proceder de un modelo que no tiene en cuenta la aportación del yeso y, por lo tanto, más sencillo que el aquí elaborado. Para prEN, la carga de rotura vendría dada por la expresión:

$$
\mathrm{P}=\lambda \sigma_{\mathrm{c}} \frac{4 \mathrm{bct}}{\mathrm{s}}
$$

